\title{
Apolipoprotein E Fragmentation within Lewy Bodies of the Human Parkinson's Disease Brain
}

\author{
Troy T Rohn* and Jacob M Mack
}

Department of Biological Sciences, Boise State University, USA

*Corresponding author: Troy T Rohn, Ph.D., Department of Biological Sciences, Boise State University, 1910 University Drive, Boise, ID 83725, USA, Tel: +208-426-2396, Fax: +208-426-1040, E-mail: trohn@boisestate.edu

\begin{abstract}
Although harboring the Apolipoprotein E4 (APOE4) allele is a well-known risk factor in Alzheimer's disease (AD), whether a similar risk holds true for Parkinson's disease (PD) is currently not known. To investigate whether apoE pathology is present in $\mathrm{PD}$, an immunohistochemical study was undertaken with fixed, human PD brain sections from the substantia nigra utilizing a recently characterized antibody that detects an amino-terminal fragment of apoE. This antibody, termed the apoE cleavage fragment p17 (nApoECFp17) antibody specifically detects an amino-terminal $17 \mathrm{kDa}$ fragment of apoE without reacting with full-length forms of the protein. Application of this antibody revealed the presence of this fragment in Lewy bodies in all cases examined. Colocalization of nApoECFp17 with an antibody to alpha-synuclein ( $\alpha-S y n)$, which served as a general marker for Lewy bodies, indicated the presence of this apoE fragment in $87.5 \%$ of all identified Lewy bodies. In addition, localization of $\mathrm{nApoECFp} 17$ was also evident within oligodendrocytes, the nucleus of melatonin-containing neurons, and blood vessels. Conversely, little staining was observed in the substantia nigra from Pick's disease or in the frontal cortex of dementia with Lewy bodies (DLB) cases, suggesting a specificity for nApoECFp 17 immunoreactivity in PD. Collectively, these data have identified widespread evidence for apoE fragmentation in the human PD brain and documented for the first time the presence of apoE within Lewy bodies, the major pathological marker for this neurodegenerative disease.
\end{abstract}

\section{Keywords}

Apolipoprotein E, Protease, Parkinson's disease, Lewy bodies, Oligodendrocytes, Nuclear localization

\section{Abbreviations}

ApoE: Apolipoprotein E; nApoECFp17: ApoE Amino-terminal Cleavage Fragment p17; PD: Parkinson's Disease; AD: Alzheimer's Disease; $\alpha-S y n:$ Alpha-synuclein; TBS: Tris-buffered Saline; DLB: Dementia with Lewy Bodies

\section{Introduction}

Parkinson's disease (PD) is the second most common age-related, progressive neurodegenerative disorder after Alzheimer's disease (AD) and is clinically characterized as a movement disorder presenting with rigidity, resting tremor, disturbances in balance and slowness in movement [1]. Pathologically, PD is characterized by the presence of intraneuronal inclusions termed Lewy bodies. Lewy bodies are circular, intracytoplasmic inclusions that contain abnormally truncated proteins, including alpha-synuclein ( $\alpha$-Syn) $[2,3]$.

Human apolipoprotein $E$ (apoE) is a polymorphic gene that includes apoE2, apoE3, and apoE4, which differ by single amino acid substitutions involving cysteine-arginine replacements at positions 112 and 158 [4]. Although inheritance of APOE4 allele is a well-known risk factor for dementia, whether it poses a similar risk in PD has yielded conflicting results (for examples see [5-12]).

We recently synthesized a site-directed cleavage antibody that specifically recognizes an amino-terminal fragment of $17 \mathrm{kDa}$ ( $p 17$ ) following cleavage after D151 of the mature, full-length form of apoE [13]. This antibody, which we termed the amino-terminal apoE cleavage fragment antibody (nApoECFp17 antibody) is highly specific for this fragment and shows no immunoreactivity to the full-length, $34 \mathrm{kDa}$ form of the protein [13]. In situ, we demonstrated widespread labeling of this antibody in the $A D$ brain and most surprising, strong localization within glia cells [13]. We further showed that a recombinantly produced fragment of apoE4, 1-151 was taken up by the microglia cell line, BV2, following extra-

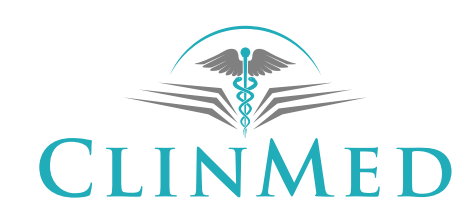

INTERNATIONAL LIBRARY

Citation: Rohn TT, Mack JM (2018) Apolipoprotein E Fragmentation within Lewy Bodies of the Human Parkinson's Disease Brain. Int J Neurodegener Dis 1:002.

Received: January 17, 2018; Accepted: February 21, 2018; Published: February 23, 2018

Copyright: (c) 2018 Rohn TT, et al. This is an open-access article distributed under the terms of the Creative Commons Attribution License, which permits unrestricted use, distribution, and reproduction in any medium, provided the original author and source are credited. 
cellular treatment and trafficked to the nucleus causing significant toxicity.

The purpose of the current study was to determine whether the presence of this fragment could also be identified in the human PD brain. Our results showed widespread immunoreactivity of nApoECFp17 in all cases examined and, for the first time, documented the presence of this amino-terminal fragment within Lewy bodies and oligodendrocytes in the substantia nigra. The presence of apoE fragmentation in postmortem PD brain sections supports the hypothesis that apoE fragmentation may be a common event, as has been previously shown in AD.

\section{Methods}

\section{Materials}

The nApoECFp17 in house, rabbit, affinity-purified polyclonal antibody has been previously characterized and is specific for the $17 \mathrm{kDa}$ amino-terminal fragment of apoE following cleavage after amino acid residue D151 [13]. This antibody does not react with full-length forms of apoE and reacts with the p17 fragment following cleavage from both full-length apoE3 and E4 [13]. The monoclonal antibody, Olig-1, was purchased from EMD Millipore (Billerica, MA). The monoclonal anti-alpha-synuclein antibody (LB 509) was purchased from Abcam (Cambridge, MA). All antibodies were used at a 1:100 dilution.

\section{Immunohistochemistry}

Autopsy brain tissue from five neuropathologically confirmed PD cases, two dementia with Lewy body (DLB) cases, and three Pick's disease cases were studied. Case demographics are presented in Table 1. Fixed substantia nigra or frontal cortex tissue sections used in this study were provided by the Institute for Memory Impairments and Neurological Disorders at the University of California, Irvine. Free-floating $40 \mu \mathrm{m}$-thick sections were used for immunohistochemical studies as previously described [14]. The tissue was provided without identifiers and thus is not considered human subject research by NIH criteria.

For single labeling, all sections were washed with 0.1 $M$ Tris-buffered saline (TBS), $\mathrm{pH} 7.4$, and then pretreat- ed with $3 \%$ hydrogen peroxide in $10 \%$ methanol to block endogenous peroxidase activity. Sections were subsequently washed in TBS with $0.1 \%$ Triton X-100 (TBS-A) and then blocked for thirty minutes in TBS-A with $3 \%$ bovine serum albumin (TBS-B). Sections were further incubated overnight at room temperature with the nApoECF antibody (1:100). Following two washes with TBS-A and a wash in TBS-B, sections were incubated in anti-rabbit or mouse biotinylated anti-IgG (1 hour) and then in avidin biotin complex (1 hour) (ABC, Elite Immunoperoxidase, Vector Laboratories, Burlingame, CA, USA). The primary antibody was visualized using Blue SG substrate (Vector Laboratories). To ensure antibody binding was specific, controls were performed that included either absence of the primary or secondary antibodies during the staining procedure. No staining was observed under these conditions.

\section{Immunofluorescence microscopy}

Immunofluorescence studies were performed by incubating sections with primary antibodies overnight at a room temperature, followed by secondary anti-rabbit or mouse biotinylated anti-IgG (1 hour) and then in $A B C$ (1 hour). Primary antibodies utilized included the nApoECFp17 (1:100), Olig-1 (mouse monoclonal, 1:100) and $\alpha$-Syn (mouse monoclonal, 1:100). For immunofluorescence co-localization studies, antigen visualization was accomplished using an Alexa fluor 488-labeled tyramide (green, $\mathrm{Ex} / \mathrm{Em}=495 / 519$ ) for one label and streptavidin Alexa fluor 555 (red, Ex/Em = 555/565) for the second label, both from Invitrogen (Carlsbad, CA). For microscopic observation and photomicrography of the Blue SG-labeled and fluorescent sections, an Olympus BX60 microscope with fluorescence capability equipped with a Magnafire SP software system for photomicrography was employed. The fluorescent molecules were excited with a $100-\mathrm{W}$ mercury lamp. Fluorescent-labeled molecules were detected using a filter set having a 460-500-nm wavelength band pass excitation filter, a 505-nm dichroic beam splitter, and a 510-560-nm band pass emission filter. To ensure that observed antibody staining was specific and not due to autofluorescence, all sections were carefully examined by fluorescence before and after labeling. The presence

Table 1: Case demographics for immunohistochemistry.

\begin{tabular}{|l|l|l|l|l|l|}
\hline Case & Sex & Age & PMI (hrs) & NPD x 1 & Cause of Death \\
\hline 1 & M & 64 & 2.25 & PD & Respiratory Failure \\
\hline 2 & M & 71 & 3.5 & PD & Cardiopulmonary Arrest \\
\hline 3 & F & 88 & 3 & PD & Pneumonia \\
\hline 4 & F & 87 & 2.3 & PD & Unknown \\
\hline 5 & M & 69 & 4.5 & PD & Cardiopulmonary Arrest \\
\hline 6 & M & 72 & 5.5 & DLB & Respiratory Failure \\
\hline 7 & M & 83 & 9.5 & DLB & Cardiopulmonary Arrest \\
\hline 8 & M & 66 & 5 & Pick's & End-stage Pick's disease \\
\hline 9 & F & 68 & 3.15 & Pick's & Acute Pulmonary Embolism \\
\hline 10 & F & 76 & 3.3 & Pick's & Cardiorespiratory Arrest \\
\hline
\end{tabular}

$\mathrm{PMI}=$ Postmortem Interval; NPD $\times 1$ = Primary pathological diagnosis . 
of Lewy bodies was only apparent following specific labeling by either the nApoECFp17 or $\alpha$-Syn antibodies.

\section{Confocal microscopy}

For confocal immunofluorescence imaging, the primary antibodies were visualized with secondary antibodies tagged with either Alexa Fluor 488 or Alexa Fluor 555 (Invitrogen, Carlsbad, CA.) Images were taken with the Zeiss LSM 510 Metasystem combined with the Zeiss Axiovert Observer Z1 inverted microscope and ZEN 2009 imaging software (Carl Zeiss, Inc., Thornwood, NY). Confocal Z-stack and single plane images were acquired with an Argon (488 nm) and a HeNe (543 nm) laser source. Z-stacks images were acquired using a 20x Plan-Apochromat (NA 0.8) objective, emission band passes of 505-550 nm for the detection of the nApoECFp17 antibody (green channel, Alexa Fluor 488) and 550-600 nm for both the detection of Olig-1 (red channel, Alexa Fluor 555) and $\alpha$-Syn (red, Alexa Fluor 555). All images displayed are 2-D maximal intensity projections generated acquired Z-stacks. Single plane images were acquired with a 63x Plan-Apochromat oil-immersion objective (NA 1.4) with emission long pass of $505 \mathrm{~nm}$ for the detection of the nApoECFp17 antibody (green channel, Alexa Fluor 488) and 550-600 $\mathrm{nm}$ for the detection of either Olig-1 or $\alpha$-Syn (red channel, Alexa Fluor 555).

\section{Statistical analysis}

To determine the percent colocalization, a quantitative analysis was performed as described previously [13] by taking $40 \mathrm{X}$ immunofluorescence, overlapping images from three different fields in the substantia nigra in three separate PD cases. Capturing was accomplished by using a $2.5 x$ photo eyepiece, and a Sony high resolution CCD video camera (XC-77). As an example, to determine the percent co-localization between nApoECFp17 and Olig-1, photographs were analyzed by counting the number of Olig-1-positive oligodendrocytes alone per 40X field for each case, and the number of cells labeled with both Olig-1 and nApoECFp17. Data are representative of the average number ( \pm S.E.M.) of each antibody alone or co-localized with both antibodies in each $40 \mathrm{X}$ field ( 3 fields total for 3 different cases). Statistical differences in this study were determined using Student's two-tailed T-test employing Microsoft Office Excel.

\section{Results}

Previous characterization of the nApoECFp17 antibody indicated that it is highly specific for a $17 \mathrm{kDa}$ amino-terminal fragment of apoE [13]. This in-house antibody recognizes the $\mathrm{N}$-terminal upstream neoepitope fragment of apoE3 and E4 that would be generated following cleavage after the terminal aspartic acid residue at position D151 of the full-length protein. Importantly, the antibody shows no reactivity to full-length forms of apoE [13]. To determine if amino-terminal fragments of apoE can be detected in the human PD brain, an immu- nohistochemical study utilizing the nApoECFp17 antibody was initiated utilizing fixed substantia nigra brain sections from confirmed PD cases. Case demographics used in this study are presented in Table 1.

As an initial step, we screened all five cases for nApoECFp17 immunoreactivity using bright-field microscopy. Following application of the nApoECFp17 antibody, widespread labeling was found in all cases examined with prominent labeling observed in apparent Lewy bodies, nuclei of melanin-containing neurons, oligodendrocytes, and along blood vessels (Figure 1). Putative staining within oligodendrocytes is based on the fact this staining was predominantly localized within white matter of substantia nigra sections and cells that were labeled displayed a linear pattern of appearance that is typical of oligodendrocytes. With the exception of Lewy body labeling, these findings are similar to what was previously documented using the nApoECFp17 antibody in the AD brain [13]. To determine whether the observed labeling of nApoECFp17 was specific to $P D$, experiments were undertaken utilizing frontal cortex sections from two DLB cases. As shown in Figure $1 \mathrm{E}$ and Figure $1 F$, we were unable to identify labeling within apparent Lewy bodies although weak labeling within glial cells was apparent (Figure $1 \mathrm{E}$ and Figure 1F, arrows). In addition, as a negative control, we also screened the substantia nigra from three Pick's disease cases. Unlike PD and DLB which are classified as pure $\alpha$-synucleinopathies, Pick's disease presents with tauopathy associated with frontotemporal lobe atrophy [15]. In all three Pick's cases, we observed a paucity of nApoECFp17 labeling with only weak glial staining in white matter (Figure $1 \mathrm{G}$ and Figure $1 \mathrm{H}$, arrows). Taken together, these data support the specificity of nApoECFp17 pathology in the substantia nigra of the human PD brain.

To confirm the presence of nApoECFp17 immunoreactivity within Lewy bodies, double-label confocal immunofluorescence studies were undertaken using $\alpha$-Syn, a standard marker for Lewy bodies. As depicted in Figure 2A, Figure 2B, Figure 2C, Figure 2D, Figure $2 \mathrm{E}$ and Figure $2 \mathrm{~F}$, Lewy bodies showed strong co-localization of these two antibodies and quantification indicated that $87.5 \%$ of identified Lewy bodies also were immunopositive for nApoECFp17 (Figure 2G).

In a previous study, a major finding was the presence of nApoECFp17 within the nuclei of glia cells of the AD brain [13]. Preliminary observations (Figure 1) suggested that a similar finding also occurs in the PD brain including possible labeling with oligodendrocytes. To confirm labeling within oligodendrocytes, double-labeling immunofluorescence confocal studies were carried out using the standard marker, Olig-1. Co-localization between Olig-1 and the nApoECFp17 antibody was observed in white matter of substantia nigra sections (Figure 3). Interestingly, while the nApoECFp17 antibody labeling was primarily nuclear, Olig-1 labeling was more widespread and appeared to label processes that 


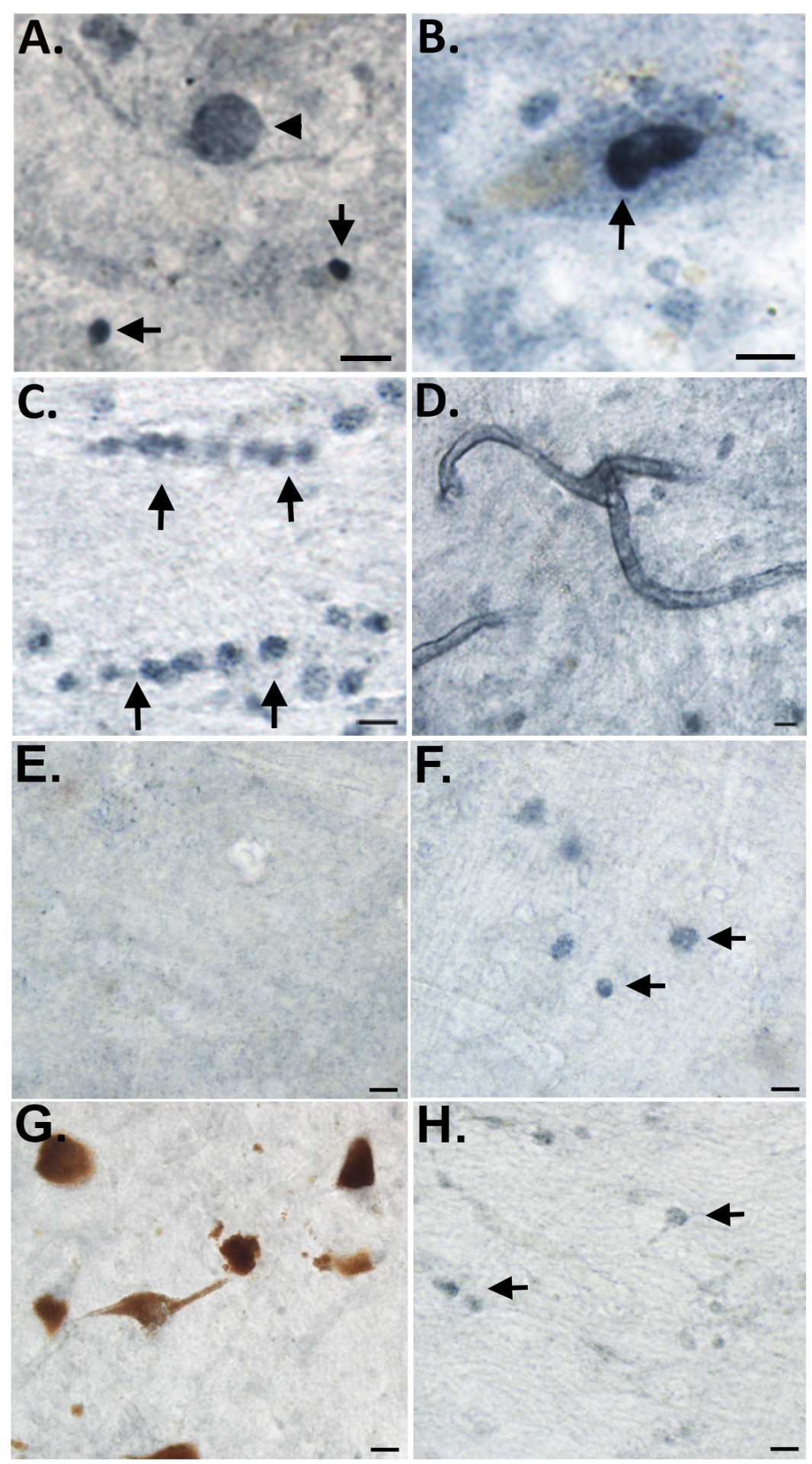

Figure 1: Detection of fragmented apoE in the substantia nigra of the PD brain. Representation bright-field staining in PD substantia nigra tissue sections following application of the nApoeCFp17 antibody (1:100) (A) Representative immunostaining of an apparent Lewy body (arrowhead) by nApoECFp17 as well as smaller circular structures (arrows); (B-D): Staining was also observed within the nucleus of melanin-containing neurons (B, arrow), in oligodendrocytes in white matter (arrows, $C$ ), and along blood vessels (D); (E and F): Representative immunostaining utilizing the nApoECFp17 (1:100) antibody in frontal cortex sections from two DLB cases indicating a relative lack of labeling except weakly within apparent oligodendrocytes in white matter $(F$, arrows); (G and $\mathbf{H})$ : Representative staining with the nApoECFp17 antibody $(1: 100)$ within the substantia nigra of two separate Pick's disease cases indicating a lack of immunoreactivity. All scale bars represent $10 \mu \mathrm{m}$.

were fragmented and damaged (Figure 3E and Figure 3F). Quantification indicated that of the total number of oligodendrocytes identified by Olig-1, $84.7 \%$ also were labeled with the nApoECFp17 antibody (Figure 3G).

\section{Discussion}

The purpose of this study was to examine whether
apoE pathology could be documented in the human PD brain. One of the major findings of the current study was the identification of this amino-terminal fragment of apoE within Lewy bodies of the PD brain. In addition, the lack of labeling of the nApoECFp17 antibody in frontal cortex of DLB cases and in the substantia nigra of Pick's disease cases suggests a specificity of nApoECFp17 pathology in 

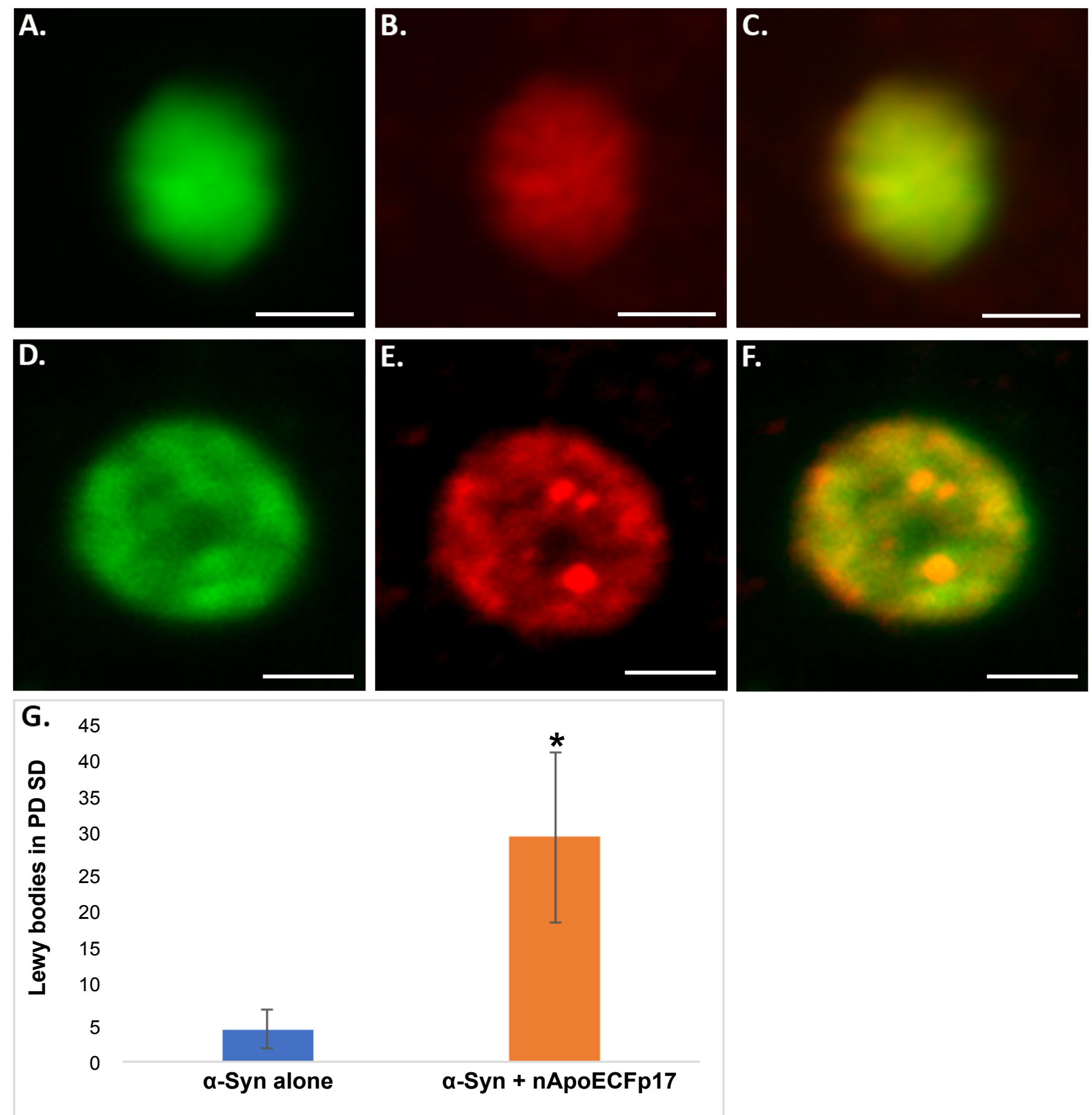

Figure 2: Localization of an amino-terminal fragment of apoE within Lewy bodies of the human PD brain (A-F): Representation images from confocal immunofluorescence in two different PD cases utilizing antibodies nApoECFp17 (A and $D$ ), $\alpha$-Syn (B and E), with the merged images shown in (C and F). Strong co-localization of the two antibodies was observed in Lewy bodies of the PD brain; (G): Quantification of the number of Lewy bodies doubled-labeled with nApoECFp17 and $\alpha-S y n$ indicated co-localization in $87.5 \%$ of the total number of Lewy bodies identified in the substantia nigra. Data depict the number of $\alpha$-Syn-labeled Lewy bodies alone (blue bar) and the number of Lewy bodies labeled with both $\alpha$-Syn and nApoECFp17 (orange bar) identified in substantia nigra PD sections by immunofluorescence microscopy $(n=3$ different $P D$ cases \pm S.D.). All scale bars represent $5 \mu \mathrm{m}$. Asterisk denotes significant difference, $p=0.018$.

PD. To our knowledge, this is the first time apoE pathology has been identified within Lewy bodies, which have been previously described as failed proteasomes containing aggregated $\alpha$-Syn and ubiquitinated proteins $[2,16]$. The presence of truncated apoE within Lewy bodies is significant as these intraneuronal structures are the major pathological markers of PD and are associated with cognitive impairment in PD and DLB $[17,18]$.

Based on the presence of truncated and aggregated proteins, including TDP-43 [19], $\alpha-S y n$, and tau [2,20], it is plausible that Lewy bodies may serve a protective function by sequestering potentially cytotoxic proteins. In the context of the current findings, previous studies have shown that amino-terminal fragments of apoE4 are cytotoxic and promote neurodegeneration [13,21,22]. Thus, apoE4 has been postulated to enhance dementia risk at the molecular level through a possible toxic-gain of function. In this manner, previous studies have shown 

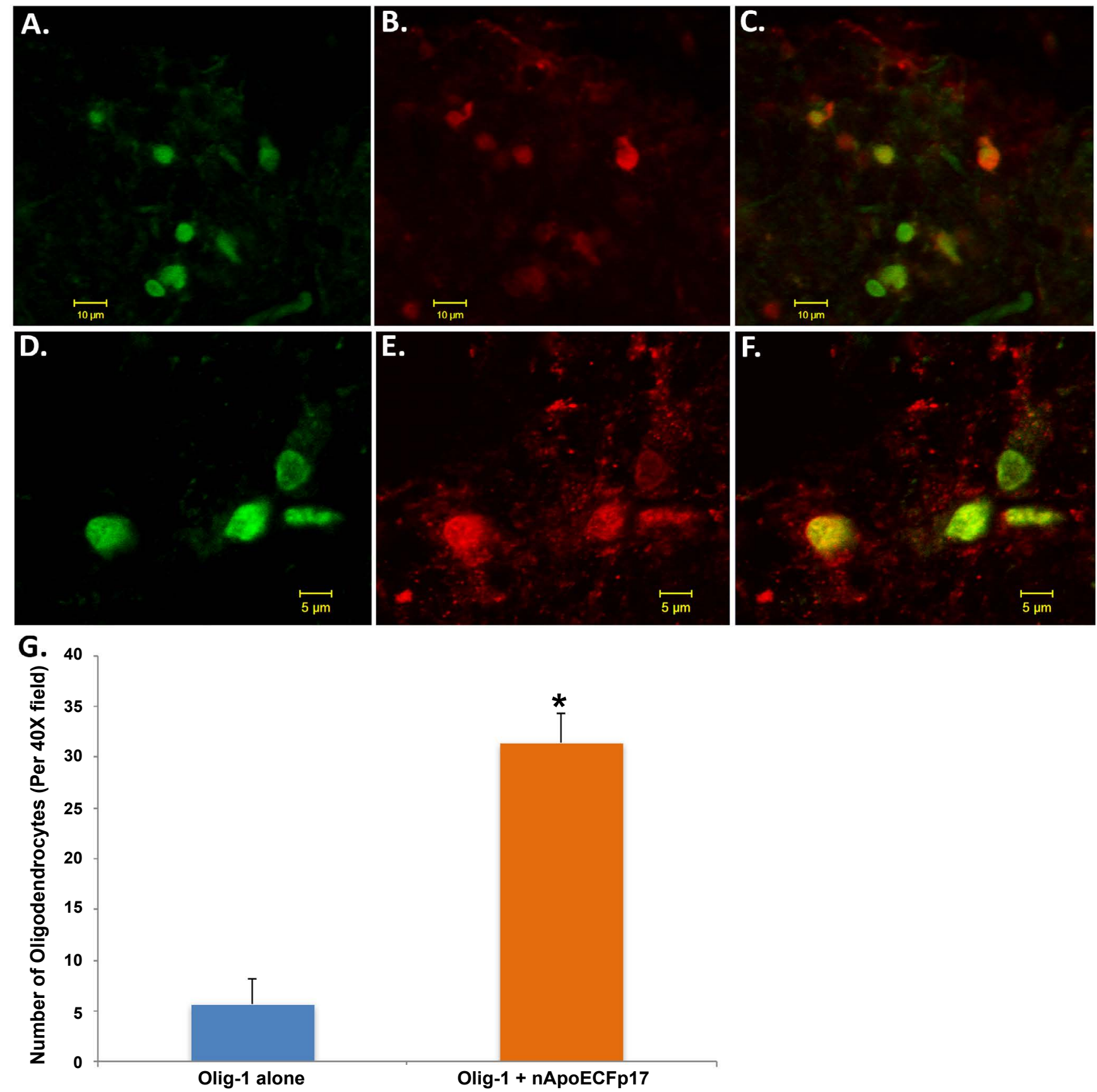

Figure 3: Localization of an amino-terminal fragment of apoE within oligodendrocytes of the human PD brain (A-F): Representation images from confocal immunofluorescence in two different PD cases utilizing antibodies nApoECFp17 (A and $D)$, Olig-1 (B and E), with the merged images shown in ( $C$ and F). Strong co-localization of the two antibodies was observed in oligodendrocytes of the PD brain; (G): Quantification of the number of oligodendrocytes doubled-labeled with nApoECFp17 and Olig-1 indicated co-localization in $84.7 \%$ of the total number of oligodendrocytes identified in respective fields. Data show the number of Olig-1-labeled-oligodendrocytes alone (blue bar) and the number of oligodendrocytes-labeled with both Olig-1 and nApoECFp17 (orange bar) identified in a 40X field within substantia nigra PD sections by immunofluorescence microscopy $\left(n=3\right.$ fields for 3 different PD cases \pm S.E.M.). Asterisk denotes significant difference, $p=1.06 \times 10^{-8}$.

that apoE4 is much more susceptible to proteolysis than apoE3 or E2 and the amino-terminal fragments that are generated are in fact neurotoxic when exposed to cultured cells or expressed in transgenic mouse models [2123]. In our recent study, we demonstrated that exposure of BV2 microglial cells to an amino-terminal fragment of apoE4 (1-151) lead to significant neurotoxicity and cell death [13]. We hypothesize that if a similar process is occurring in the PD brain as documented by the presence of nApoECFp17, this could promote the underlying neu- rodegeneration associated with this disease. In addition, cognitive impairment is prevalent in PD, affecting 15$20 \%$ of patients [24], and recent studies have supported APOE4 allele carrier frequency was significantly higher in PD with executive dysfunction $[25,26]$. Therefore, apoE4 may also contribute to cognitive impairment associated with PD as previously suggested $[27,28]$. Our current results demonstrating the presence of fragmented apoE adds to the growing body of studies implicating this protein in the pathogenesis of PD. 
The accumulation of aggregated forms of $\alpha$-Syn protein into Lewy bodies is one of the characteristic features of PD [2]. A question raised from the current findings is: What is the potential significance between $\alpha$-Syn and nApoECF (1-151) detected by our nApoECFp17 antibody? In a study by Emamzadeh, et al., the authors examined the effects of different isoforms of apoE on aggregation of $\alpha$-Syn and found that aggregation is stimulated by all isoforms, with apoE4 showing the greatest stimulatory effect [29]. Based on our documentation of apoE pathology in the PD brain, together these studies suggest that low levels of apoE may seed $\alpha$-Syn aggregation, which could potentially lead to a pathway of $\alpha$-Syn-induced neurodegeneration.

An additional finding of this study was the presence of truncated apoE within oligodendrocytes in white matter of the PD brain. Interesting, many of these labeled oligodendrocytes appeared to display fragmented processes (Figure 3E and Figure 3F), an indication that these cells may be undergoing degeneration. Because of the critical role oligodendrocytes play in myelination of axons in the CNS, the degeneration of oligodendrocytes may contribute to the extrapyramidal symptoms associated with PD.

It is important to point out a couple of caveats of the current study. First, we were unable to obtain information on specific APOE4 allelic genotype for the PD cases used in this study. Therefore, we were unable to dissect out any possible influence of harboring the APOE 4 allele on our findings. Second, the primary antibody used in this study, nApoECFp17, specifically immunolabels a 17 $\mathrm{kDa}$ amino-terminal fragment of apoE without reacting to full-length forms of the protein [13]. Because apoE2, $E 3$, and E4 differ by single amino acid substitutions involving cysteine-arginine replacements at positions 112 and 158 [4], the nApoECFp17 does not discriminate between the different isoforms and will readily recognize the $\mathrm{p} 17$ fragment derived from full-length apoE3 and E4 [13].

\section{Conclusion}

The presence of truncated apoE as shown in the present study supports the notion that these species are being sequestered within Lewy bodies. However, whether truncated apoE in PD is a cause or effect of the disease will require further studies. Ideally, a comprehensive study that would include examining specific APOE4 allelic cases would need to be undertaken to examine whether fragmentation happens to a greater degree in $A P O E 4$ carriers.

\section{Acknowledgements}

This work was funded by National Institutes of Health Grant 1R15AG042781-01A1. Funding was also supported by the KO Alzheimer's Dementia Foundation (Boise, ID). The project described was supported by Institu- tional Development Awards (IDeA) from the National Institute of General Medical Sciences of the National Institutes of Health under Grants \#P20GM103408 and P20GM109095. The funders had no role in study design, data collection and analysis, decision to publish, or preparation of the manuscript.

\section{References}

1. Tofaris GK, Spillantini MG (2005) Alpha-synuclein dysfunction in Lewy body diseases. Mov Disord 20: S37-S44.

2. Spillantini MG, Schmidt ML, Lee VM, Trojanowski JQ, Jakes R, et al. (1997) Alpha-synuclein in Lewy bodies. Nature 388: 839-840.

3. Spillantini MG, Crowther RA, Jakes $R$, Hasegawa $M$, Goedert M (1998) alpha-Synuclein in filamentous inclusions of Lewy bodies from Parkinson's disease and dementia with lewy bodies. Proc Natl Acad Sci U S A 95: 6469-6473.

4. Weisgraber KH, Rall SC Jr, Mahley RW (1981) Human E apoprotein heterogeneity. Cysteine-arginine interchanges in the amino acid sequence of the apo-E isoforms. J Biol Chem 256: 9077-9083.

5. Pavlova R, Mehrabian S, Petrova M, Skelina S, Mihova K, et al. (2014) Cognitive, neuropsychiatric, and motor features associated with apolipoprotein $E \varepsilon 4$ allele in a sample of Bulgarian patients with late-onset Parkinson's disease. Am J Alzheimers Dis Other Demen 29: 614-619.

6. Multhammer $M$, Michels $A$, Zintl $M$, Mendoza $M C$, Klunemann HH (2014) A large ApoE $\varepsilon 4 / \varepsilon 4$ homozygous cohort reveals no association with Parkinson's disease. Acta Neurol Belg 114: 25-31.

7. Li YJ, Hauser MA, Scott WK, Martin ER, Booze MW, et al. (2004) Apolipoprotein $E$ controls the risk and age at onset of Parkinson disease. Neurology 62: 2005-2009.

8. Pulkes T, Papsing C, Mahasirimongkol S, Busabaratana M, Kulkantrakorn K, et al. (2011) Association between apolipoprotein E genotypes and Parkinson's disease. J Clin Neurosci 18: 1333-1335.

9. Khan N, Graham E, Dixon P, Morris C, Mander A, et al. (2001) Parkinson's disease is not associated with the combined alpha-synuclein/apolipoprotein E susceptibility genotype. Ann Neurol 49: 665-668.

10. Kurz MW, Dekomien G, Nilsen OB, Larsen JP, Aarsland D, et al. (2009) APOE alleles in Parkinson disease and their relationship to cognitive decline: A population-based, longitudinal study. J Geriatr Psychiatry Neurol 22: 166-170.

11. Eerola J, Launes J, Hellstrom O, Tienari PJ (2002) Apolipoprotein E (APOE), PARKIN and catechol-O-methyltransferase (COMT) genes and susceptibility to sporadic Parkinson's disease in Finland. Neurosci Lett 330: 296-298.

12. Huertas I, Jesus S, Garcia-Gomez FJ, Lojo JA, Bernal-Bernal I, et al. (2017) Genetic factors influencing frontostriatal dysfunction and the development of dementia in Parkinson's disease. PLoS One 12: e0175560.

13. Love JE, Day RJ, Gause JW, Brown RJ, Pu X, et al. (2017) Nuclear uptake of an amino-terminal fragment of apolipoprotein E4 promotes cell death and localizes within microglia of the Alzheimer's disease brain. Int J Physiol Pathophysiol Pharmacol 9: 40-57.

14. Rohn TT, Catlin LW (2011) Immunolocalization of influenza $A$ virus and markers of inflammation in the human Parkinson's disease brain. PLoS One 6: e20495. 
15. Uchihara T, Ikeda K, Tsuchiya K (2003) Pick body disease and Pick syndrome. Neuropathology 23: 318-326.

16. McNaught KS, Olanow CW, Halliwell B, Isacson O, Jenner $P(2001)$ Failure of the ubiquitin-proteasome system in Parkinson's disease. Nat Rev Neurosci 2: 589-594.

17. Hurtig HI, Trojanowski JQ, Galvin J, Ewbank D, Schmidt ML, et al. (2000) Alpha-synuclein cortical Lewy bodies correlate with dementia in Parkinson's disease. Neurology 54 1916-1921.

18. Mattila PM, Rinne JO, Helenius H, Dickson DW, Roytta M (2000) Alpha-synuclein-immunoreactive cortical Lewy bodies are associated with cognitive impairment in Parkinson's disease. Acta Neuropathol 100: 285-290.

19. Kokoulina P, Rohn TT (2010) Caspase-cleaved transactivation response DNA-binding protein 43 in Parkinson's disease and dementia with Lewy bodies. Neurodegener Dis 7: 243-250.

20. Ishizawa T, Mattila P, Davies P, Wang D, Dickson DW (2003) Colocalization of tau and alpha-synuclein epitopes in Lewy bodies. J Neuropathol Exp Neurol 62: 389-397.

21. Huang $Y$, Liu XQ, Wyss-Coray $T$, Brecht WJ, Sanan DA, et al. (2001) Apolipoprotein $E$ fragments present in Alzheimer's disease brains induce neurofibrillary tangle-like intracellular inclusions in neurons. Proc Natl Acad Sci U S A 98: 8838-8843.

22. Harris FM, Brecht WJ, Xu Q, Tesseur I, Kekonius L, et al. (2003) Carboxyl-terminal-truncated apolipoprotein E4 causes Alzheimer's disease-like neurodegeneration and behavioral deficits in transgenic mice. Proc Natl Acad Sci U S A 100: 10966-10971.

23. Brecht WJ, Harris FM, Chang S, Tesseur I, Yu GQ, et al. (2004) Neuron-specific apolipoprotein e4 proteolysis is associated with increased tau phosphorylation in brains of transgenic mice. J Neurosci 24: 2527-2534.

24. Riedel O, Klotsche J, Spottke A, Deuschl G, Forstl H, et al. (2008) Cognitive impairment in 873 patients with idiopathic Parkinson's disease. Results from the German Study on Epidemiology of Parkinson's Disease with Dementia (GEPAD). J Neurol 255: 255-264.

25. Samat NA, Abdul Murad NA, Mohamad K, Abdul Razak MR, Mohamed Ibrahim N (2017) Apolipoprotein Eع4: A Biomarker for Executive Dysfunction among Parkinson's Disease Patients with Mild Cognitive Impairment. Front Neurosci 11: 712.

26. Mata IF, Leverenz JB, Weintraub D, Trojanowski JQ, Hurtig $\mathrm{HI}$, et al. (2014) APOE, MAPT, and SNCA genes and cognitive performance in Parkinson disease. JAMA Neurol 71: 1405-1412.

27. Huang X, Chen P, Kaufer DI, Troster AI, Poole C (2006) Apolipoprotein $E$ and dementia in Parkinson disease: A meta-analysis. Arch Neurol 63: 189-193.

28. Morley JF, Xie SX, Hurtig HI, Stern MB, Colcher A, et al. (2012) Genetic influences on cognitive decline in Parkinson's disease. Mov Disord 27: 512-518.

29. Emamzadeh FN, Aojula H, McHugh PC, Allsop D (2016) Effects of different isoforms of apoE on aggregation of the a -synuclein protein implicated in Parkinson's disease. Neurosci Lett 618: 146-151. 